\title{
Bidirectional Higher Order Cascaded Raman Amplification Benefits for 10-Gb/s WDM Unrepeated Transmission Systems
}

\author{
Stefano Faralli, Gabriele Bolognini, Giovanni Sacchi, Simone Sugliani, and Fabrizio Di Pasquale, Member, IEEE
}

\begin{abstract}
Benefits provided by higher order bidirectional Raman pumping schemes in $10-\mathrm{Gb} / \mathrm{s}$ unrepeated wavelengthdivision-multiplexing transmission systems are experimentally quantified in terms of BER performances at $10 \mathrm{~Gb} / \mathrm{s}$. By keeping under control double-Rayleigh-scattering-noise-induced transmission penalties, which can degrade system performance at very high ON-OFF Raman gain, as well as nonlinear propagation effects such as Brillouin scattering, self- and cross-phase modulations, four-wave-mixing, and Raman-induced crosstalks, we show a total unrepeated system reach enhancement up to $3.5 \mathrm{~dB}$ with respect to first-order bidirectional pumping. As confirmed by theory, the maximum reach enhancement is mainly limited by pump-to-signal relative intensity noise transfer induced by higher order copumping.
\end{abstract}

Index Terms-Optical communication systems, Raman amplifiers, Rayleigh scattering, relative intensity noise (RIN).

\section{INTRODUCTION}

$\mathbf{T}$ ECHNOLOGIES for extended-reach unrepeated wavelength-division-multiplexing (WDM) transmission systems are attracting a great deal of attention due to possible system cost reduction. Forward-error correction (FEC) techniques [1] and distributed Raman amplification [2] have been demonstrated to be effective in implementing this goal. In particular, higher order Raman pumping schemes have been used for lowering the distributed amplifier equivalent optical noise figure $\left(\mathrm{NF}_{\mathrm{EQ}}\right)$ and for cascaded pump delivery to remote amplifiers [3], [4]. In both cases, higher order configurations provide benefits in terms of maximum unrepeated system reach [about 2.4-dB enhancement in the 1480-nm pump power delivered to remote erbium-doped fiber amplifiers (EDFAs) and up to 2.5-dB improvement in the optical equivalent noise figure]. Important issues requiring careful consideration include signal double-Rayleigh-scattering (DRS) noise, which can induce transmission penalties at high counterpropagating ON-OFF Raman gains $\left(G_{\mathrm{ON}-\mathrm{OFF}}\right)$ [5], and pump-to-signal relative intensity noise (RIN) transfer [6], which is critical when considering copumping schemes.

Although signal DRS noise enhancement in higher order counterpumping schemes has been studied theoretically [5] and

Manuscript received December 21, 2004; revised March 23, 2005.

S. Faralli, G. Bolognini, and F. Di Pasquale are with Scuola Superiore Sant'Anna di Studi Universitari e Perfezionamento, 56123 Pisa, Italy (e-mail: stefano.faralli@cnit.it).

G. Sacchi and S. Sugliani are with Laboratorio Nazionale di Reti Fotoniche, CNIT, 56123 Pisa, Italy (e-mail: giovanni.sacchi@ cnit.it).

Digital Object Identifier 10.1109/JLT.2005.850807 also observed experimentally [4], to the best of the authors' knowledge, no transmission experiments have been reported to quantitatively evaluate DRS-noise-induced transmission penalties at high ON-OFF Raman gain $\left(G_{\mathrm{ON}-\mathrm{OFF}}\right)$.

In this paper, we first consider counterpumping configurations and compare the equivalent optical noise figure $\left(\mathrm{NF}_{\mathrm{EQ}}\right)$, signal-to-DRS-noise ratio $\left(\mathrm{OSNR}_{\mathrm{DRS}}\right)$ and bit error rate (BER) performance at $10 \mathrm{~Gb} / \mathrm{s}$ of first- and third-order counterpropagating Raman pumping schemes.

In particular, the impact of DRS noise enhancement in the two pumping configurations is quantified, as a function of $G_{\mathrm{ON}-\mathrm{OFF}}$, by measuring the OSNR required at the receiver site in order to guarantee a given BER value. An OSNR penalty ( $\triangle$ OSNR) for each ON-OFF Raman gain can also be defined as the difference between the two OSNR values respectively required for a given BER with third- and first-order pumping schemes.

Once the equivalent optical noise figure has been optically characterized as a function of $G_{\mathrm{ON}-\mathrm{OFF}}$, the penalty $\Delta$ OSNR allows one to quantify the effective span length increase, provided by third-order counterpumping with respect to first order, without additional transmission penalties.

This approach allows one to identify optimum ON-OFF Raman gain values for best performance in long-span unrepeated WDM transmission systems, keeping DRS-noiseinduced transmission penalties under control.

We then experimentally compare the transmission performances of bidirectional configurations based on first- and second-order copumping schemes, with fixed and optimized third-order counterpumping Raman schemes.

In other words, we separately quantify, by BER measurements at $10 \mathrm{~Gb} / \mathrm{s}$, benefits provided respectively by higher order co- and counterpumping schemes, showing a total maximum reach enhancement up to $3.5 \mathrm{~dB}$ in a single span, compared with standard first-order bidirectional pumping. We also identify sources of $Q$-factor penalties, which limit these benefits, mainly related to pump-to-signal RIN transfer, induced by the secondorder copumping configuration [6].

\section{EXPERIMENTAL SETUP}

Fig. 1 describes the experimental setup used for the $10-\mathrm{Gb} / \mathrm{s}$ BER measurement in long-span unrepeated WDM transmission systems.

Seven 100-GHz-spaced distributed feedback (DFB) lasers (ITU grid from 1550.1 to $1554.9 \mathrm{~nm}$ ) are multiplexed, 


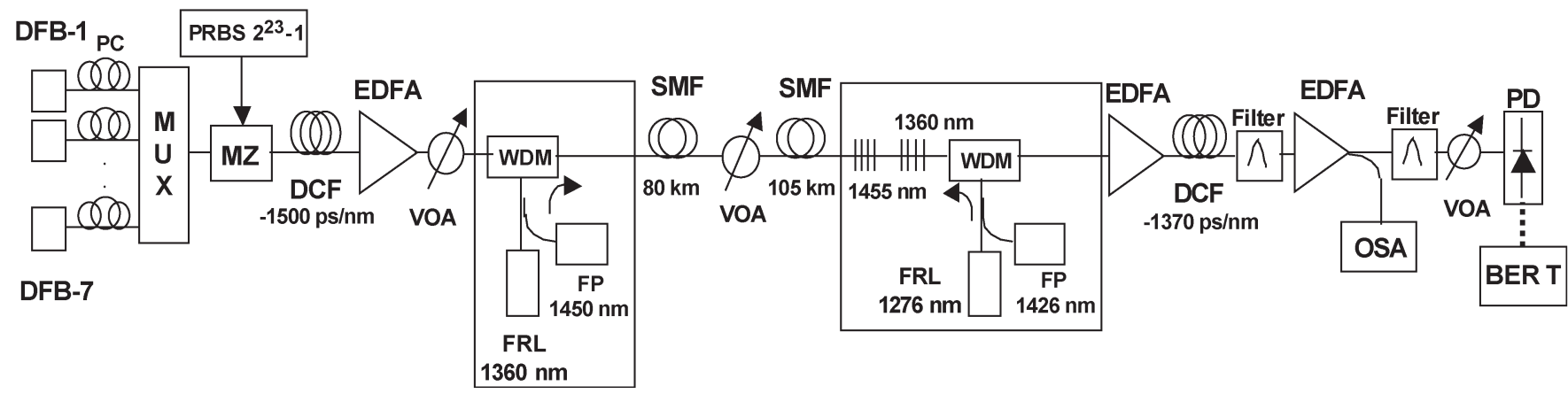

Fig. 1. Experimental setup for BER measurement in higher order bidirectionally pumped Raman amplifiers.

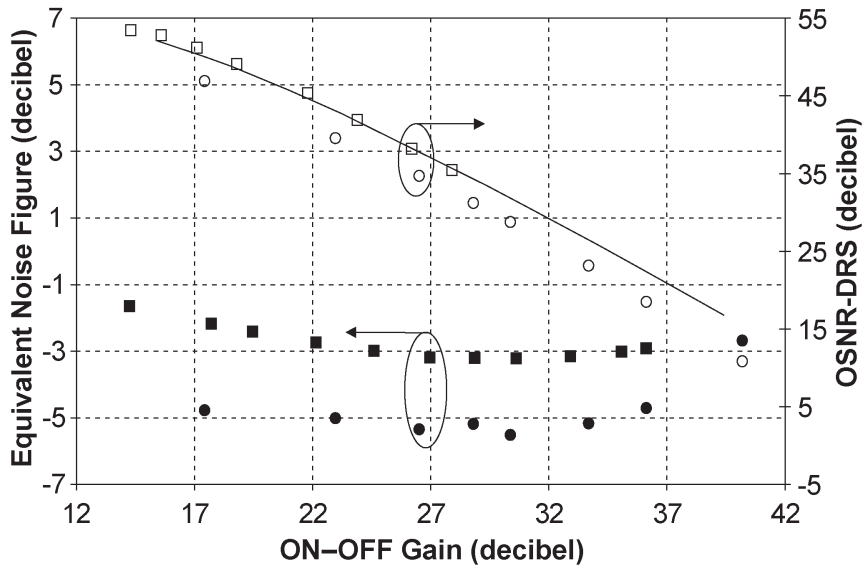

Fig. 2. Equivalent optical noise figure (solid symbols) and signal-to-DRSnoise ratio (open symbols) versus $G_{\mathrm{ON}-\mathrm{OFF}}$ for first- (squares) and thirdorder pumping (circles) schemes at $1554.9 \mathrm{~nm}$. Also, computed DRS noise ratio for first-order pumping scheme (line).

modulated at $9.953 \mathrm{~Gb} / \mathrm{s}$ [pseudorandom binary sequence (PRBS) $2^{23}-1$, NRZ format] through a Mach-Zender modulator (MZ), decorrelated by a dispersion-compensating fiber [(DCF): $-1500 \mathrm{ps} / \mathrm{nm}$ ], which also provides precompensation, before being amplified and coupled into $185 \mathrm{~km}$ of standard single-mode fiber (SSMF); two fiber spools are used with a middle variable optical attenuator (VOA) for controlling the total span loss $L$. A bidirectional higher order Raman pumping configuration is also described in Fig. 1. In particular, while the second-order copropagating scheme is quite simple, based on a high-power fiber Raman laser (FRL) at $1360 \mathrm{~nm}(1.5 \mathrm{~W})$, combined with two polarization-multiplexed Fabry-Pérot (FP) lasers at $1450 \mathrm{~nm}$, the third-order counterpropagating configuration is more complex. A very-high-power (up to $3.5 \mathrm{~W}$ ) FRL at $1276 \mathrm{~nm}$ is combined with a 1426-nm seed, generated by a depolarized laser diode; in addition, an amplified fiber cavity is formed by two high-reflectivity fiber Bragg gratings at 1360 and $1455 \mathrm{~nm}$ and low-reflectivity distributed Rayleigh scattering mirrors. The 1426-nm seed, launched with the primary pump in counterpropagating direction, is amplified as it propagates along the SSMF, reaching its maximum value well inside the fiber spool, consequently reducing the distributed amplifier equivalent optical noise figure up to $2.5 \mathrm{~dB}$ [3]. By adjusting the 1276-nm pump and 1455-nm seed powers, a Raman gain flatness better than $2 \mathrm{~dB}$ can be ensured over the whole $C$-band (1530-1565 nm) up to $G_{\mathrm{ON}-\mathrm{OFF}} \sim 28 \mathrm{~dB}$.
A seed at $1480 \mathrm{~nm}$ could be similarly introduced, providing about 2.4-dB enhancement in the pump power delivered to remote EDFAs [4].

Note that the DRS noise contribution, which would be introduced by adding more SMFs instead of using a middle span VOA, is negligible, as the two fiber spools are longer than the Raman amplifier effective length.

At the receiver side, the WDM channels are selected by tunable filters and amplified by a dual-stage EDFA including an additional DCF for chromatic dispersion compensation $(-1370 \mathrm{ps} / \mathrm{nm})$. A VOA allows one to obtain a constant optical power at the photodetector. BER measurements are carried out varying the decision threshold; the minimum BER at optimum threshold is obtained by extrapolation [7].

\section{Higher Order COUNTERPUMPING: OpticAL PERFORMANCE AND TRANSMISSION EXPERIMENT}

We have first studied higher order counterpumping schemes and performed a single-channel transmission experiment without copumping, in order to identify the maximum system reach enhancement provided by third order with respect to first-order counterpumping. The first-order pump is provided by a singlewavelength $(1450 \mathrm{~nm})$ high-power $(1.5 \mathrm{~W})$ FRL (not shown in Fig. 1).

For the sake of simplicity in this first set of experiments, we only consider the 105-km-long SSMF spool with chromatic dispersion compensation at the transmitter side $(-1500 \mathrm{ps} / \mathrm{nm})$. This allows us to concentrate our attention on DRS noise penalties induced by counterpropagating Raman pumping, avoiding all potential degradation induced by nonlinear propagation effects within the first $80-\mathrm{km}$ fiber spool. BER performance versus received OSNR is measured by simply varying the VOA loss at the transmitter EDFA output.

We have first studied the optical performance of the two pumping schemes: Fig. 2 compares the measured $\mathrm{NF}_{\mathrm{EQ}}$ and OSNR $_{\text {DRS }}$ for first- and third-order pumping schemes versus $G_{\text {ON-OFF }}$ at $1555 \mathrm{~nm}$.

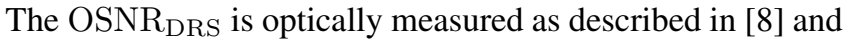
[9] by directly modulating the tunable laser at the amplifier input and using two acousto-optical modulators at the output in order to ensure a high extinction ratio and a consequently high-sensitivity measurement (up to $55 \mathrm{~dB}$ ). We observe an equivalent optical noise figure reduction of about $2 \mathrm{~dB}$ in the case of third-order pumping, which is however associated with 


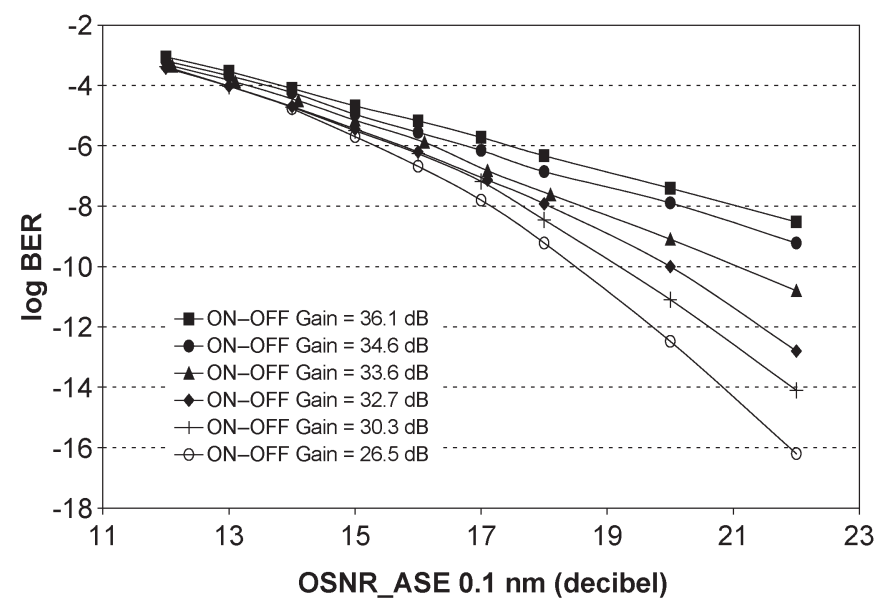

(a)

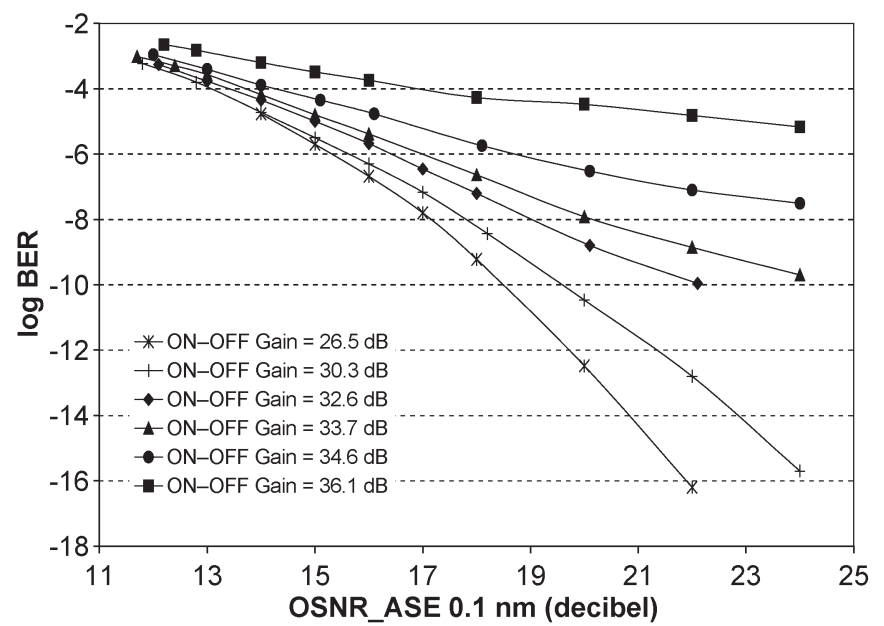

(b)

Fig. 3. BER versus OSNR at different $G_{\mathrm{ON}-\mathrm{OFF}}$ for (a) first-order pumping and (b) third-order pumping.

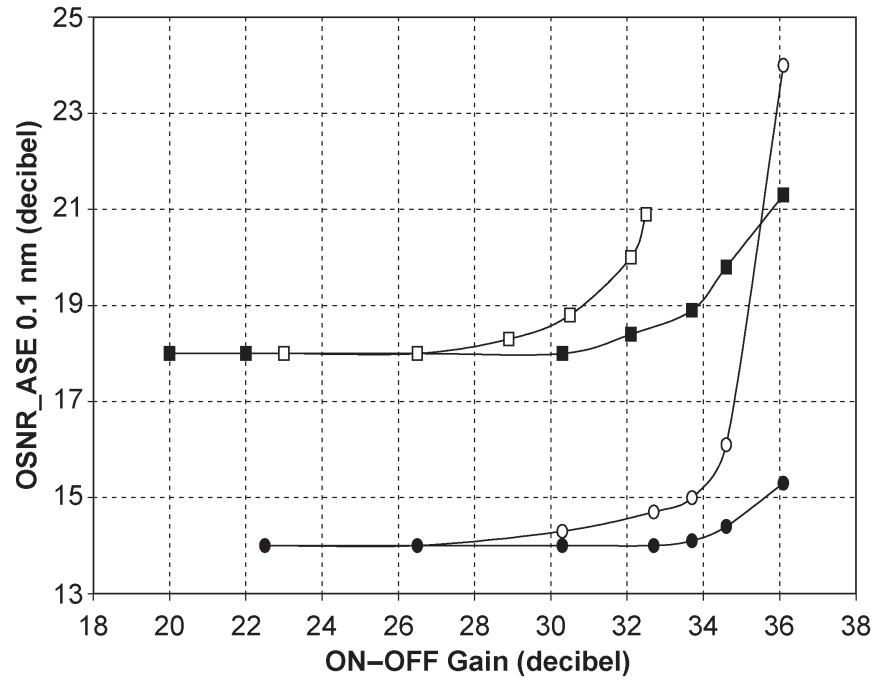

Fig. 4. OSNR values versus $G_{\mathrm{ON}-\mathrm{OFF}}$ required at the receiver in order to guarantee BER values of $10^{-5}$ (circles) and $10^{-9}$ (squares) for first- (solid symbols) and third-order pumping (open symbols) schemes at $1554.9 \mathrm{~nm}$.

a DRS noise enhancement. Also note that at $G_{\mathrm{ON}-\mathrm{OFF}}$ greater than about $35 \mathrm{~dB}$, the $\mathrm{NF}_{\mathrm{EQ}}$ performance of both pumping schemes is degraded by amplified spontaneous emission (ASE) single-Rayleigh backscattering.

Although a $Q$-factor analysis can be used to quantify the relative impact of DRS-induced multipath interference and ASE noises [5], in this paper we follow an experimental approach based on BER measurements. In order to quantify potential transmission penalties related to this DRS noise enhancement and identify the optimum $G_{\mathrm{ON}-\mathrm{OFF}}$ for penaltyfree transmission, we have first measured BER versus OSNR at different ON-OFF Raman gains for first- and third-order pumping schemes [see Fig. 3(a) and (b)], and then evaluated the OSNR values versus $G_{\mathrm{ON}-\mathrm{OFF}}$, required in both configurations in order to ensure fixed BER values of $2 \times 10^{-9}$ and $2 \times 10^{-5}$ (Fig. 4). The OSNR at the receiver side is simply varied by changing the VOA loss at the transmitter EDFA output.
Note that the OSNR penalty $(\triangle \mathrm{OSNR})$ of the third-order pumping scheme with respect to first-order pumping strongly depends on the BER values and only becomes significant at extremely high $G_{\mathrm{ON}-\mathrm{OFF}}$ when considering an uncorrected BER of $2 \times 10^{-5}$. However, a more sensible uncorrected BER value of $10^{-9}$ (pre-FEC) should be considered in order to guarantee adequate system margins; in this case, transmission penalties related to DRS noise enhancement can be kept lower than $0.5 \mathrm{~dB}$ by limiting the maximum $G_{\mathrm{ON}-\mathrm{OFF}}$ to less than about $30 \mathrm{~dB}$.

As the received OSNR at the EDFA output depends mainly on the distributed Raman amplifier $\mathrm{NF}_{\mathrm{EQ}}$, its value does not increase significantly for $G_{\mathrm{ON}-\mathrm{OFF}}$ greater than about $25 \mathrm{~dB}$. As a consequence, the benefits provided by higher order Raman counterpumping schemes in terms of optical noise figure reduction can be fully exploited without any DRS-noise-induced transmission penalty. On the other hand, when considering cascaded pump delivery for remote EDFA pumping, one could try to enhance the delivered pump at $1480 \mathrm{~nm}$ even more by further increasing $G_{\mathrm{ON}-\mathrm{OFF}}$. From Fig. 4, we can see that this would induce significant OSNR penalties for gains greater than $30 \mathrm{~dB}$ and would cancel out the benefits already provided by the 2.4-dB-higher 1480-nm pump delivered at the remote EDFA [4].

We have finally performed a transmission experiment in order to identify the maximum span loss achievable with firstand third-order Raman counterpumping schemes.

The $80-\mathrm{km}$ SSMF spool has been introduced at the transmitter side with additional chromatic dispersion compensation $(-1370 \mathrm{ps} / \mathrm{nm})$ at the receiver between two EDFA stages, as shown in Fig. 1. A VOA is used between the two fiber spools to control the total span loss.

A DFB laser at $1554.9 \mathrm{~nm}$ is externally modulated at $10 \mathrm{~Gb} / \mathrm{s}$ (PRBS $2^{23}-1$, NRZ format), and BER values are measured versus OSNR at different $G_{\mathrm{ON}-\mathrm{OFF}}$. A maximum average signal power of $10 \mathrm{dBm}$ has been coupled into the transmission fiber in order to avoid transmission penalties in the first part of the span, due to nonlinear propagation effects such as Brillouin scattering and self-phase modulation (the received OSNR is 


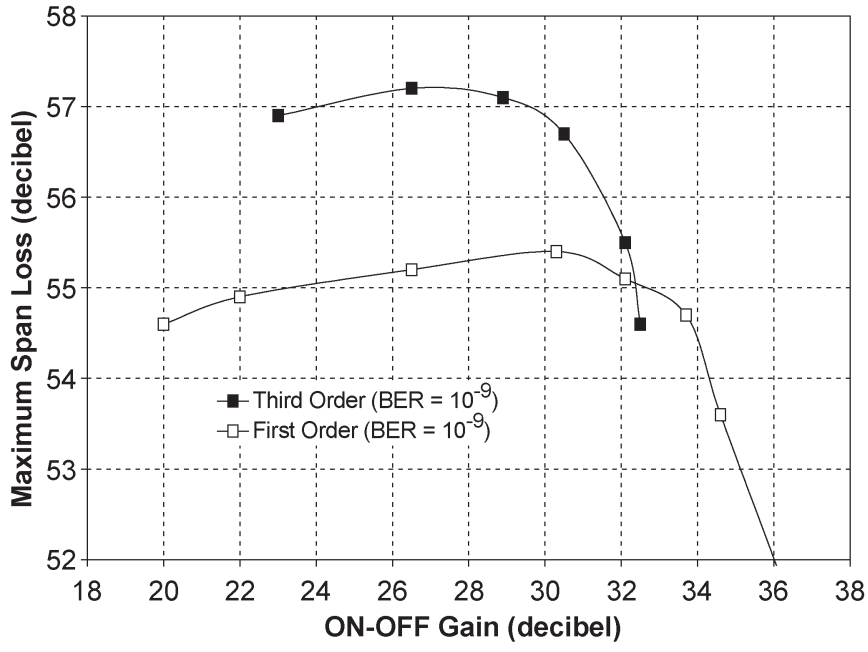

Fig. 5. Maximum span loss for first- and third-order pumping schemes versus $G_{\mathrm{ON}-\mathrm{OFF}}\left(\mathrm{BER}=10^{-9}\right)$.

now varied changing the span losses by the middle VOA). The chromatic dispersion compensation map has been designed in order to keep under control impairments induced by self-phase modulation.

Fig. 5 compares the maximum span loss achievable with firstand third-order counterpumping schemes versus $G_{\mathrm{ON}-\mathrm{OFF}}$, considering a required uncorrected BER value of $10^{-9}$.

We see that the maximum tolerable span loss for third-order pumping is $\sim 2 \mathrm{~dB}$ greater than the best achievable with first-order pumping and occurs at a lower $G_{\mathrm{ON}-\mathrm{OFF}}$, about $27 \mathrm{~dB}$ compared to the optimum $G_{\mathrm{ON}-\mathrm{OFF}}$ of $\sim 30 \mathrm{~dB}$ for first-order pumping. With further increases in gain, penalties due to DRS noise degrade the third-order pumping scheme performance until finally, at $G_{\mathrm{ON}-\mathrm{OFF}}=32 \mathrm{~dB}$, its advantage over first-order pumping disappears. At even higher gain values, both first- and third-order pumping schemes are penalized by a combination of ASE light single-Rayleigh backscattering and signal DRS noise.

The observations that Rayleigh-scattering-induced penalties begin to appear at lower $G_{\mathrm{ON}-\mathrm{OFF}}$ for higher order pumping schemes and that DRS noise starts to degrade system performance at lower gains than ASE light single backscattering confirm the theoretical results presented in [5].

\section{Bidirectional Higher Order Pumping: WDM TRANSMISSION EXPERIMENT}

Keeping the third-order counterpumping configuration fixed, with an average counterpropagating gain of $\sim 27 \mathrm{~dB}$ in order to avoid DRS-noise-induced transmission penalties, in this section we compare the transmission performances introducing firstand second-order copumping schemes. First-order copumping is implemented by polarization multiplexed two high-power FP lasers, which can couple up to $700 \mathrm{~mW}$ pump power at $1450 \mathrm{~nm}$ into the transmission fiber. The second-order copumping scheme has already been described in Fig. 1 and combines a high-power FRL at $1360 \mathrm{~nm}$ and a seed at $1450 \mathrm{~nm}$. As shown in Fig. 1, we are now considering seven 100-GHz-spaced channels (setup already described in Section II).

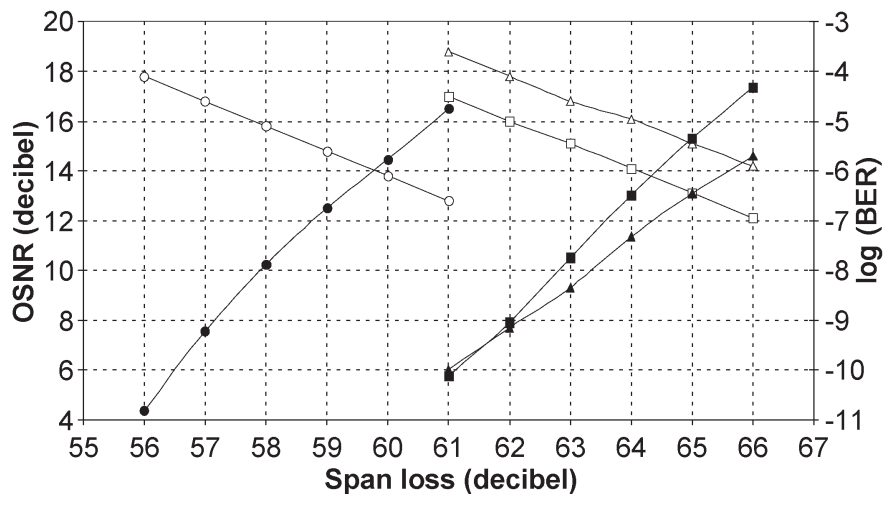

Fig. 6. BER (solid symbols) and OSNR (open symbols) versus span loss for third-order counterpumping scheme (circles), first-order copump and thirdorder counterpump (squares), second-order copump, and third-order counterpump (triangles).

Note that in order to ensure improved performances and to avoid nonlinear propagation effects such as Brillouin scattering, self-phase modulation, cross-phase modulation, four-wavemixing crosstalk, and Raman-induced cross-gain modulation (XGM), copumping schemes require a careful optimization of both pump power and signal power per channel launched into the transmission fiber.

Furthermore, the pump RIN must be sufficiently low in order to avoid penalties induced by noise transfer from pump to signals. In case of first-order copumping, the best configuration for maximum OSNR improvement without additional transmission penalties corresponds to quite a low signal power per channel $(-2 \mathrm{dBm})$ and high copropagating ON-OFF Raman gain $(16 \mathrm{~dB})$, with limited gain saturation (less than $3 \mathrm{~dB}$ ) to avoid Raman XGM [10]. Note that, with such a high Raman gain, using higher signal power per channel will induce transmission penalties, mainly due to stimulated Brillouin scattering.

Comparing BER and OSNR performances at $1554.9 \mathrm{~nm}$ versus span loss, with and without first-order copumping, points out a maximum system reach enhancement up to $5 \mathrm{~dB}$ (BER $=$ $10^{-9}$ ), as shown in Fig. 6.

With fixed BER $=10^{-9}$, the corresponding OSNR values suggest a beneficial interplay between chromatic dispersion and nonlinear propagation effects in case of first-order copumping.

Finally, we have investigated second-order copumping, keeping the optimized third-order counterpumping configuration fixed.

By coupling the maximum available second-order pump power at $1360 \mathrm{~nm}(1.25 \mathrm{~W})$, and optimizing the 1450-nm FP seed and channel input signal powers $(70 \mathrm{~mW}$ and $-2 \mathrm{dBm}$, respectively), only a slight improvement of maximum span reach $(\sim 0.6 \mathrm{~dB})$ has been observed at uncorrected BER $=$ $10^{-9}$ with respect to first-order copumping. In addition, when using second-order copumping schemes, the same BER performances as in first-order copumping are achieved at higher OSNR values. All these features point out an increased source of penalty for second-order copumping, which is likely related to RIN transfer from the 1360 -nm pump to the WDM signals, through the seed at $1450 \mathrm{~nm}$, as suggested in [6]. Fig. 7(a) and (b) shows the eye patterns at $1554.9 \mathrm{~nm}$ for the two pumping 


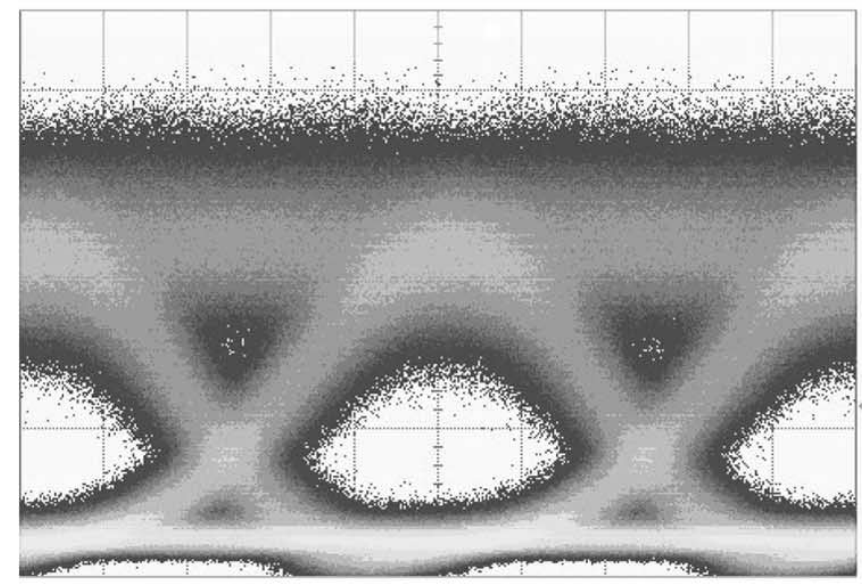

(a)

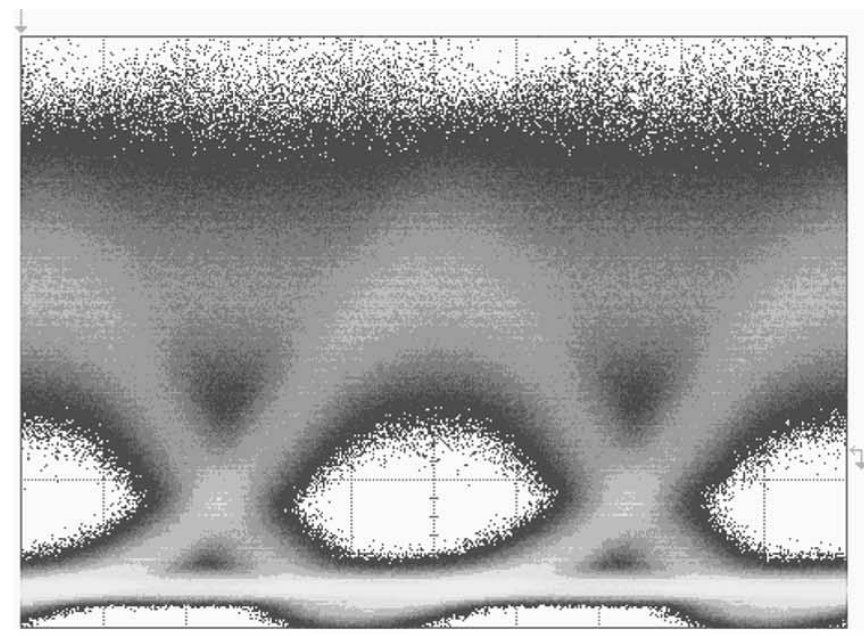

(b)

Fig. 7. Eye diagram at $1554.9 \mathrm{~nm}$ with (a) first-order copumping and (b) second-order copumping.

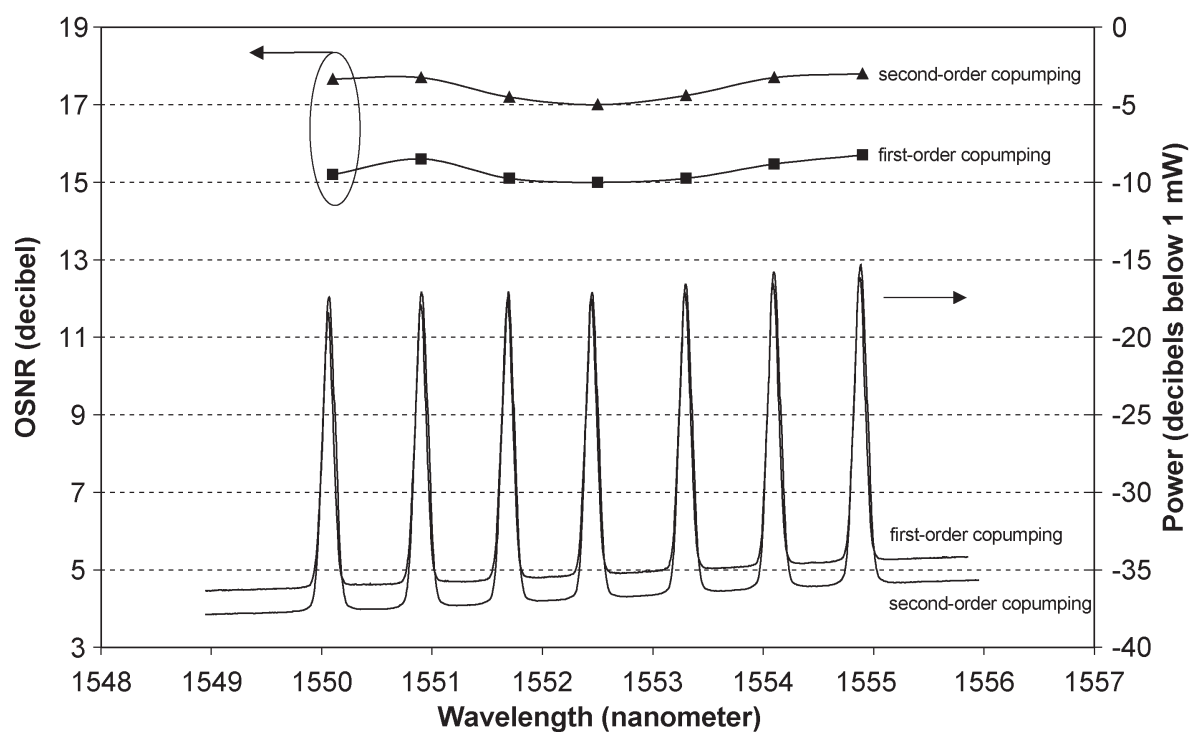

Fig. 8. Output spectra and OSNR for optimum first- and second-order copumping schemes $(L=62 \mathrm{~dB})$.

schemes at BER $\sim 10^{-9}$, confirming a visible degradation the mark levels in case of second-order copumping.

On the other hand, BER and OSNR performances versus span loss at $1554.9 \mathrm{~nm}$, also reported in Fig. 6 for this configuration, show that an improved system reach of about $1.5 \mathrm{~dB}$ can only be achieved at a higher uncorrected BER (i.e., at $\mathrm{BER}=10^{-6}$ ), thus requiring enhanced FEC techniques; in these conditions the lower required OSNR values reduce the impact of transmission penalties.

Fig. 8 shows the output spectra and OSNR performance (0.1-nm resolution bandwidth) for all seven channels in case of optimum first- and second-order copumping schemes (span loss $L=62 \mathrm{~dB}$, BER $\sim 10^{-9}$ in both schemes). An OSNR improvement of about $2 \mathrm{~dB}$ in second-order copumping is evident. In Fig. 9, the measured $Q$ factors for all seven channels are shown, along with the corresponding $Q$ penalties with respect to back-to-back configuration at the same OSNR level (ASE noise loading), for both first- and second-order copumping schemes, with fixed third-order counterpumping configuration (span loss $62 \mathrm{~dB}$, corresponding to BER $\sim 10^{-9}$ ). It is evident from the graph that second-order copumping provides almost the same transmission performance as first-order copumping, but with much higher $Q$ penalties; this is likely due to pump-tosignal RIN transfer.

\section{Bidirectional Higher Order Pumping: $Q$ FACTOR PENALTY}

In order to theoretically investigate the source of penalties affecting higher order copumping and verify the experimental results previously shown, we have computed the $Q$ penalty induced by second-order pump-to-signal RIN transfer (with respect to first-order copumping $Q$ factors) for all the WDM channels [11], in the same configuration of Fig. 9.

Using the model proposed in [11], the calculated cutoff frequency $\omega_{\mathrm{c} 1}$ for RIN transfer from the FRL $(1360 \mathrm{~nm})$ to the FP pump $(1450 \mathrm{~nm})$ is $\omega_{\mathrm{c} 2}=20 \mathrm{MHz}$, while $\omega_{\mathrm{c}}=8 \mathrm{MHz}$ for RIN transfer from FP pump (1450 nm) to WDM channels 


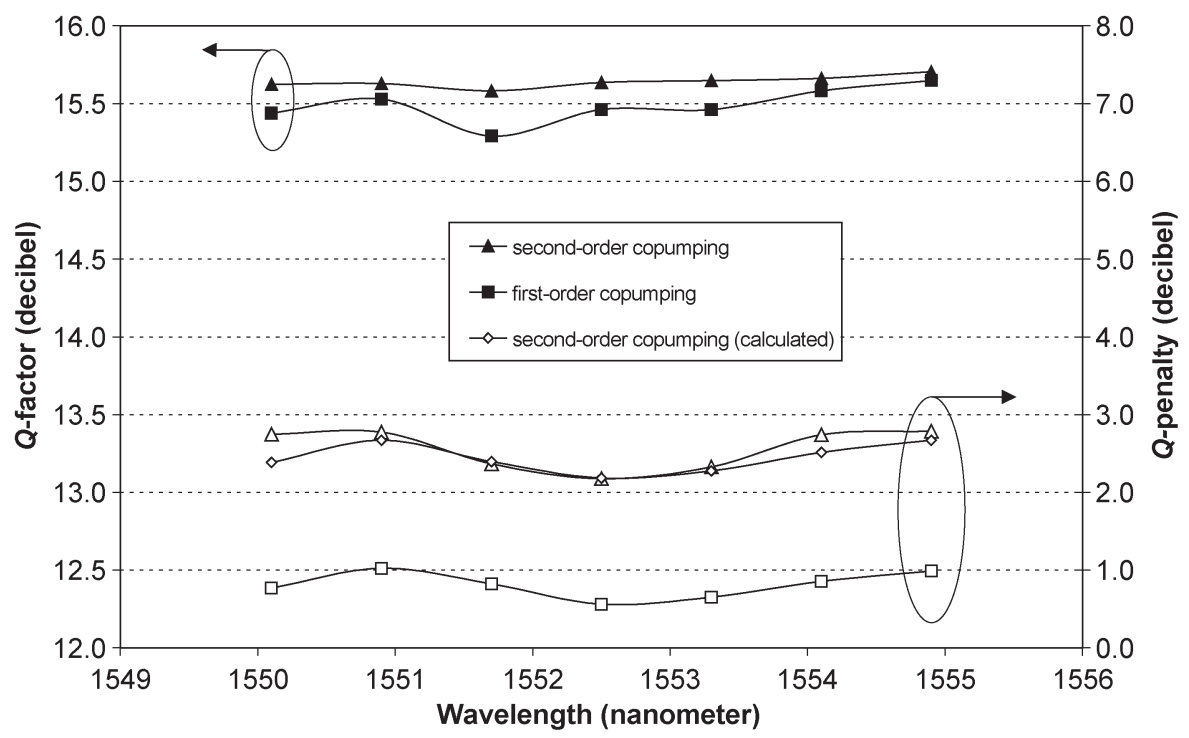

Fig. 9. Experimental $Q$-factor (solid symbols) and $Q$ penalty (open symbols) for first- (squares) and second-order (triangles) copumping schemes, and theoretical $Q$ penalty for second-order copumping (open diamonds) for WDM channels as in Fig. 8.

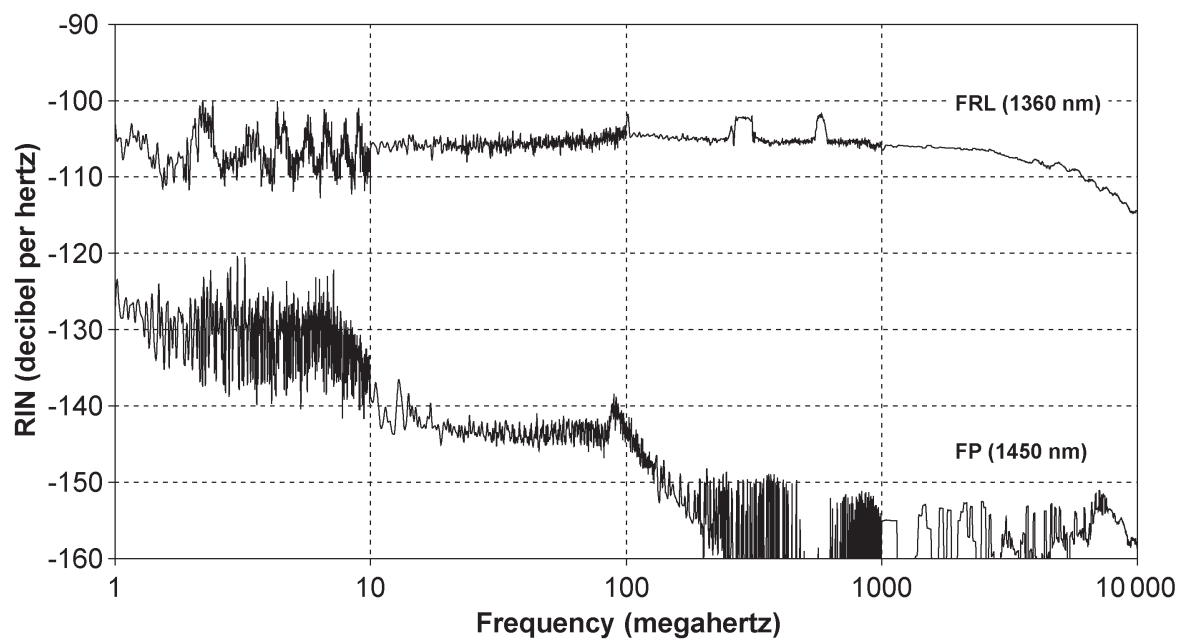

Fig. 10. RIN values for second-order pump (FRL at $1360 \mathrm{~nm}$ ) and first-order pump (FP at $1450 \mathrm{~nm}$ ).

(around $1550 \mathrm{~nm}$ ). Note that these cutoff frequencies strongly depend on fiber chromatic dispersion and its slope.

In order to accurately describe RIN-induced $Q$ factor penalties, measured data for pump RIN down to low frequencies are required as input data of the model. Therefore, the RIN values of the first- and second-order pumps have been measured at the amplifier input from $10 \mathrm{kHz}$ to $10 \mathrm{GHz}$ and are shown in Fig. 10.

The RIN is transferred from the second-order pump to the WDM channels at around $1550 \mathrm{~nm}$, through the first-order pump.

The induced penalty has been computed for each channel by using the equation [11]

$$
Q_{\text {penalty }}=\sqrt{1+Q_{\mathrm{b}}^{2} \cdot \mathrm{XT}_{\mathrm{RIN}}}
$$

where $Q_{\mathrm{b}}$ is the $Q$ factor with first-order copumping, and $\mathrm{XT}_{\mathrm{RIN}}$ is the integral of the signal $\operatorname{RIN}(f)$ over the receiver bandwidth.
Note that the RIN-induced crosstalk and the corresponding $Q$ penalty increase for higher RIN transfer cutoff frequencies.

The computed penalty is about $1.6 \mathrm{~dB}$ with respect to firstorder pumping, which is in good agreement with the experimental results, as shown in Fig. 9, confirming RIN transfer to be the supposed origin of high $Q$ penalties in second-order copumping.

Calculations also pointed out that in our case the first-order pump input RIN has a minor impact on system penalties, since the amount of RIN transferred from second- to first-order pump during Raman amplification is much higher than the input firstorder pump RIN (the input RIN crosstalk of first-order pump is $\mathrm{XT}_{\mathrm{RIN}}=-40 \mathrm{~dB}$, while the total crosstalk of RIN transferred to first-order pump is $\mathrm{XT}_{\mathrm{RIN}}=-24 \mathrm{~dB}$ ).

Therefore, lowering the first-order pump RIN would result in negligible system penalty reduction, while simulations for our case indicated that it would be possible to decrease $Q$ penalty of second-order copumping to less than $0.2 \mathrm{~dB}$ (with respect to first-order) by using a second-order copump with RIN values less than $-120 \mathrm{~dB} / \mathrm{Hz}$. 


\section{CONCLUSION}

In conclusion, experiments showed that higher order bidirectional Raman pumping in unrepeated wavelength-divisionmultiplexing (WDM) transmission systems offers sensible benefits in terms of optical signal-to-noise ratio (OSNR) improvement and span reach enhancement; existing penalties are mainly induced by higher order copumping. Theory and experiment confirm that the maximum unrepeated system reach enhancement is currently limited by pump-to-signal relative intensity noise (RIN) transfer penalties, and hence could be further increased by optimizing the higher order copropagating pump in order to ensure lower RIN values.

\section{ACKNOWLEDGMENT}

The authors would like to thank MPB Communications, Canada, for providing the third-order counterpropagating pump unit and Marconi Communications, Italy, for helpful discussions and support.

\section{REFERENCES}

[1] O. A. Sab, "FEC techniques in submarine transmission systems," presented at the Optical Fiber Communication (OFC), Anaheim, CA, 2001, Paper TuF1-3.

[2] L. Labrunie, F. Boubal, P. Le Roux, and E. Brandon, "500 Km WDM $12 \times 10 \mathrm{~Gb} / \mathrm{s}$ CRZ repeaterless transmission using second order remote amplification," Electron. Lett., vol. 39, no. 19, pp. 1394-1395, 2003.

[3] S. B. Papernyi, V. I. Karpov, and W. R. L. Clements, "Third order cascaded Raman amplification," presented at the Proc. Optical Fiber Communication (OFC), Anaheim, CA, 2002, Postdeadline Paper FB4-1.

[4] V. Karpov, S. Papernyi, V. Ivanov, and W. Clements, "Cascaded pump delivery for remotely pumped erbium doped fiber amplifiers," presented at the Suboptic, Monte-Carlo, Monaco, 2004, Poster Paper We 8.8.

[5] S. Faralli and F. Di Pasquale, "Impact of double Rayleigh scattering noise in distributed higher order Raman pumping schemes," IEEE Photon. Technol. Lett., vol. 15, no. 6, pp. 804-806, Jun. 2003.

[6] M. D. Mermelstein, K. Brar, and C. Headley, "RIN transfer suppression technique for dual-order Raman pumping schemes," IEEE Photon. Technol. Lett., vol. 15, no. 10, pp. 1354-1356, Oct. 2003.

[7] N. Bergano, F. W. Kerfoot, and C. R. Davidson, "Margin measurement in optical amplifier systems," IEEE Photon. Technol. Lett., vol. 5, no. 3, pp. 304-306, Mar. 1993.

[8] S. A. E. Lewis, S. V. Chernikov, and J. R. Taylor, "Characterization of double Rayleigh scattering noise in Raman amplifiers," IEEE Photon. Technol. Lett., vol. 12, no. 5, pp. 528-530, May 2000.

[9] G. Bolognini, S. Sugliani, and F. Di Pasquale, "Double Rayleigh scattering noise in Raman amplifiers using pump time-division-multiplexing schemes," IEEE Photon. Technol. Lett., vol. 16, no. 5, pp. 1286-1288, May 2004

[10] T. Yamamoto and S. Norimatsu, "Statistical analysis on stimulated Raman crosstalk in dispersion-managed fiber links," J. Lightw. Technol., vol. 21, no. 10, pp. 2229-2239, Oct. 2003.

[11] C. R. S. Fludger, V. Handerek, and R. J. Mears, "Pump to signal RIN transfer in Raman fiber amplifiers," J. Lightw. Technol., vol. 19, no. 8, pp. 1140-1148, Aug. 2001.

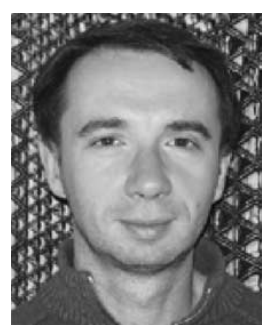

Stefano Faralli was born in Siena, Italy. He received the B.S. degree in physics from the University of Pisa, Pisa, Italy, in 2000, and the Master's degree in optical communications systems and networks from the Politecnico di Milano, Milan, Italy, in 2001.

$\mathrm{He}$ is a Postdoctoral Fellow at the Scuola Superiore Sant'Anna, Pisa, Italy. His current research interests at the National Excellence Center in Photonic Networks, Scuola Superiore Sant'Anna, include Raman amplifiers, erbium-doped fiber and waveguide amplifiers, and their applications in wavelengthdivision-multiplexing communication systems and networks.

Gabriele Bolognini, photograph and biography not available at the time of publication.

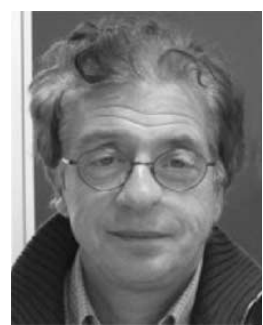

Giovanni Sacchi was born in Milan, Italy, in 1949. $\mathrm{He}$ received the B.S. degree in physics from the University of Milan, Italy, in 1979.

From 1980 to 1997, he worked for Italtel, Milan, Italy, as a Photonic Components Manager. From 1997 to 2000, he worked for Pirelli Cavi, Milan, Italy, as Optical Amplifiers Designer. From 2000 to 2002, he was with Cisco Photonics, Monza, Italy, working as Senior Researcher. Since 2002, he has been a Researcher at CNIT (Consorzio Nazionale Interuniversitario per le Telecomunicazioni), Pisa, Italy. He has filed ten international patents, and he is the author and coauthor of more than 20 scientific journal and conference papers in the area of optical amplifiers and optical communications systems. His research interests are in the area of optical amplifiers and fiber optic transmissions.

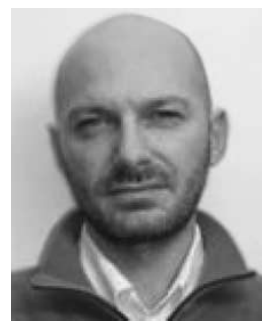

Simone Sugliani was born in Bergamo, Italy, in 1972. He received the B.S. degree in materials engineering from Politecnico di Milano, Milan, Italy, in 1999 and the Master's degree in optical systems and networks from Politecnico di Milano in 2001.

Since 2002, he has been a Research Assistant at the National Photonic Networks laboratory (CNIT), Pisa, Italy. His current research interests cover the area of optical amplification and fiber-optic systems.

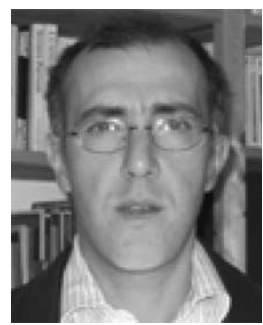

Fabrizio Di Pasquale (M'03) was born in Italy in 1963. He received the B.S. degree in electronic engineering from the University of Bologna, Italy, in 1989 and the Ph.D. degree in information technology from the University of Parma, Italy, in 1993.

From 1993 to 1998, he was with the Department of Electrical and Electronic Engineering, University College London, U.K., as a Research Fellow, working on optical amplifiers, wavelength-divisionmultiplexing (WDM) optical communication systems, and liquid-crystal displays. After two years with Pirelli Cavi e Sistemi and two years with Cisco Photonics Italy, he is now Associate Professor in Telecommunications at the Scuola Superiore Sant'Anna, Pisa, Italy. His current research interests include Raman amplifiers, erbiumdoped fiber amplifiers, and their applications in WDM communication systems and networks. He has filed 15 international patents, and he is the author and coauthor of more than 60 scientific journals and conference papers in the area of optical amplifiers, optical communications systems, and liquid-crystal displays. 